\title{
Comunicación
}

\section{Extracción incidental y reimplantación de vejiga urinaria en un canino con piometra en el hospital médico veterinario ASMEVET. Reporte de caso}

\author{
Incidental extraction and reimplantation of the urinary bladder in a canine with \\ pyometra at the Veterinary Medical Hospital ASMEVET. Case report
}

\author{
Luis E. Chávez ${ }^{1,4}$, César A. Pérez², Rincón Karol³, María Santamaría ${ }^{3}$, \\ Néstor Laverde ${ }^{2}$
}

\section{Resumen}

El propósito de este reporte de caso es presentar y documentar una complicación quirúrgica en una perra de raza Beagle de 4 años, a la cual se le seccionó la vejiga a la altura del cuello durante una celiotomía exploratoria, realizada para corregir un piometra de muñón originada en el momento de una ovariohisterectomía selectiva. La paciente fue remitida al Hospital Médico Veterinario ASMEVET de la ciudad de Tunja, Boyacá, Colombia, con historia de vómito, depresión y disuria días después del procedimiento quirúrgico. En el examen físico se evidenció aumento de la temperatura rectal, postración y dolor a la palpación del abdomen. Se tomaron muestras para cuadro hemático y química sanguínea para evaluar función renal y hepática. Además, se practicó una ecografía abdominal. Los diagnósticos diferenciales fueron obstrucción iatrogénica de uréteres,

\footnotetext{
${ }^{1}$ Facultad de Ciencias Agropecuarias, Universidad Pedagógica y Tecnológica de Colombia, Tunja, Colombia

${ }^{2}$ Fundación Universitaria Juan De Castellanos, Facultad de Ciencias Agrarias y Ambientales, Tunja, Colombia

${ }^{3}$ Universidad Agraria de La Habana, Cuba

${ }^{4}$ E-mail: luisevet1137@yahoo.es
}

Recibido: 28 de octubre de 2020

Aceptado para publicación: 20 de abril de 2021

Publicado: 24 de agosto de 2021

CLos autores. Este artículo es publicado por la Rev Inv Vet Perú de la Facultad de Medicina Veterinaria, Universidad Nacional Mayor de San Marcos. Este es un artículo de acceso abierto, distribuido bajo los términos de la licencia Creative Commons Atribución 4.0 Internacional (CC BY 4.0) [https:// creativecommons.org/licenses/by/4.0/deed.es] que permite el uso, distribución y reproducción en cualquier medio, siempre que la obra original sea debidamente citada de su fuente original 
piometra cervical, peritonitis por cuerpo extraño e íleo paralitico. Se hizo una celiotomía donde se evidenció un absceso y secuestro de vísceras circundantes. En el intento de separarlas se seccionó la vejiga a nivel del cuello. Esta fue reimplantada con un patrón de sutura Gambee y complementada con omentalización. La paciente recuperó el control de micción.

Palabras clave: vejiga urinaria, piometra, uréteres, reimplantación

\section{Abstract}

This report presents and documents a surgical complication in a 4-year-old Beagle dog, whose bladder was sectioned at the neck level during an exploratory celiotomy performed to correct a stump pyometra originated at the time of a selective ovariohysterectomy. The patient was referred to the ASMEVET Veterinary Medical Hospital in the city of Tunja, Boyacá, Colombia, with a history of vomiting, depression and dysuria days after the surgical procedure. Physical examination revealed increased rectal temperature, prostration, and pain on palpation of the abdomen. Samples were taken for a blood count and blood chemistry to evaluate kidney and liver function. In addition, an abdominal ultrasound was performed. Differential diagnoses were iatrogenic ureter obstruction, cervical pyometra, foreign body peritonitis, and paralytic ileus. A celiotomy was performed where an abscess and sequestration of the surrounding viscera were evidenced. In the attempt to separate them, the bladder was sectioned at the level of the neck. This was reimplanted with a Gambee suture pattern and supplemented with omentalization. The patient regained urination control.

Key words: urinary bladder, pyometra, ureters, reimplantation

\section{INTRODUCCIÓN}

La ovariohisterectomía (OVH) es reconocida por ser uno de los procedimientos quirúrgicos más realizados en medicina veterinaria, por ser el método de elección de control de población y enfermedades infecciosas reproductivas aplicadas a caninos. Además, es un procedimiento relativamente sencillo, determinado que las complicaciones asociadas a la cirugía sean comunes, tales como hemorragia, piometra de muñón, abscesos, incontinencia urinaria, traumatismo ureteral, hernia incisional (Muraro y White, 2014), aunque también se ha reportado torsión de la vejiga debido a una colocación incorrecta durante el cierre de la pared abdominal (Thieman y Pozzi, 2010).
La ruptura de la vejiga urinaria es una complicación común en perros y gatos asociada a trauma, inflamaciones severas, cistocentesis, cateterización, o compresión manual durante el examen clínico o laparotomía (Lipscomb, 2018). La ruptura de vejiga es un inconveniente que debe ser corregido de inmediato para evitar las complicaciones asociadas al uroperitoneo como son: peritonitis, uremia, acidosis y síndrome compartimental (Gaut y Ortiz, 2014).

Se reporta el caso de una complicación quirúrgica en una perra de raza Beagle, a la cual se le seccionó la vejiga a la altura del cuello durante una celiotomía exploratoria realizada para corregir un piometra de muñón posterior a una ovariohisterectomía. 


\section{Caso Clínico}

\section{Anamnesis}

Se presenta a consulta en el Hospital Médico Veterinario ASMEVET en la ciudad de Tunja, Boyacá, Colombia, un canino hembra, de raza Beagle, de 4 años, con historia de vómito, depresión y dificultad a la hora de orinar. El propietario informa que la paciente había sido sometida a esterilización selectiva, donde le informaron que debido al sobrepeso de la paciente fue imposible realizar la extirpación completa de las estructuras uterinas, de manera que deciden reprogramar la cirugía para un mes después.

\section{Examen Clínico}

Al examen clínico presentó los siguientes hallazgos anormales: temperatura rectal: $39.8^{\circ} \mathrm{C}$, postración, dolor a la palpación abdominal, frecuencia cardiaca $120 \mathrm{lpm}$. Como diagnósticos diferenciales se tuvieron en consideración una obstrucción iatrogénica de uréteres, piometra cervical, peritonitis por cuerpo extraño e íleo paralitico.

\section{Análisis Clínico y de Laboratorio}

Se tomaron muestras de sangre para determinar cuadro hemático, perfil hepático (fosfatasa alcalina - ALP, aspartato aminotransferasa - AST), y renal (creatinina, nitrógeno ureico en sangre - BUN). El cuadro hemático mostró leucocitosis, con desviación a la izquierda y disminución de las proteínas plasmáticas. En la química sanguínea hubo aumento del BUN.

Se decidió realizar ecografía abdominal, en la cual se evidenció una imagen irregular y anecogénica compatible con un absceso a nivel de la región prepúbica. Debido a su gran tamaño no se logró determinar el origen, por lo que se decidió realizar laparotomía exploratoria.

\section{Tratamiento Quirúrgico}

Al realizar la laparotomía exploratoria se observó en el abdomen caudal y cavidad pélvica un absceso de gran tamaño con adherencias, secuestro de vísceras no diferenciables. Asimismo, en membranas serosas se observaron cambios compatibles con peritonitis. Durante el procedimiento se buscó eliminar las adherencias, el pus y liberar las estructuras comprometidas con ayuda de tijeras tipo Metzembaund. En esta maniobra se seccionó accidentalmente la vejiga urinaria a nivel del cuello, separándola del cuerpo (Figura 1), el pliegue genito-vesical y los uréteres.

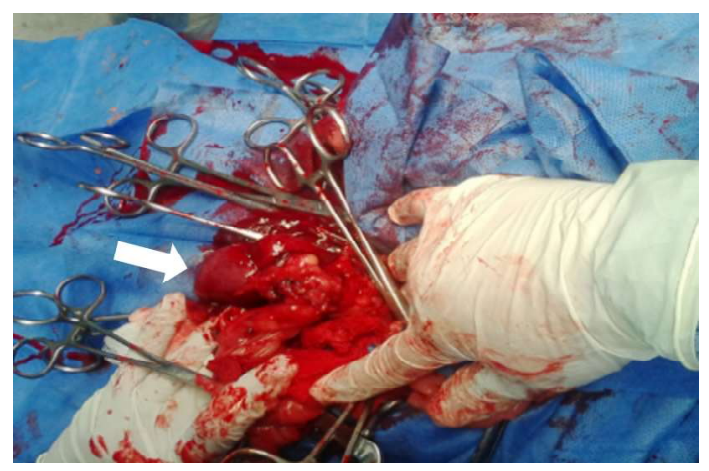

Figura 1. Vejiga urinaria de perra Beagle fuera de la cavidad abdominal al ser seccionada durante una laparotomía exploratoria (flecha)

Al ser seccionados por accidente y extraídos de la cavidad abdominal las estructuras anatómicas mencionadas, se decide reimplantar los uréteres a la vejiga y esta al cuello vesical con patrones de Gambee (Figura 2) y complementar con omentalización.

Se fijó una sonda uretral para evitar la excesiva distención de la vejiga urinaria por 10 días buscando una adecuada fijación de los tejidos adosados (Figura 3). Pasados los 


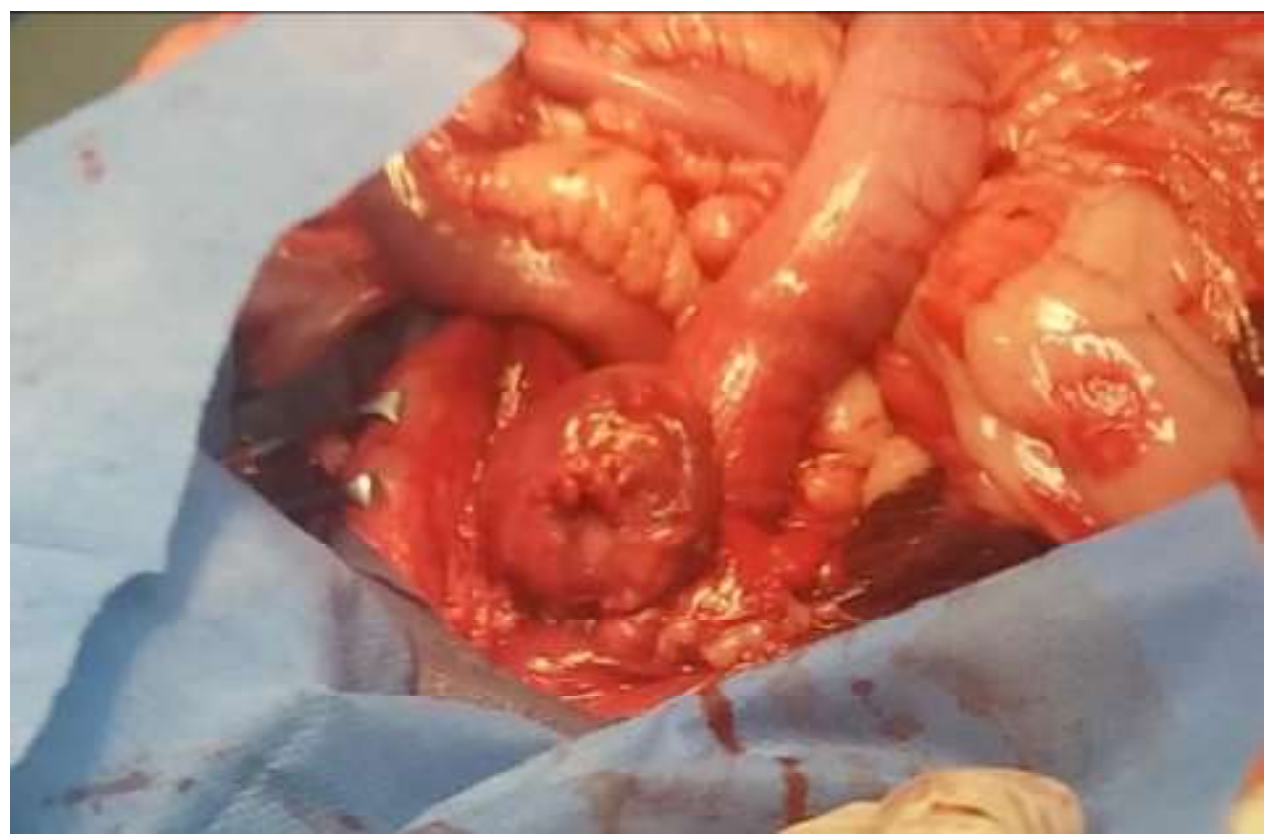

Figura 2. Vejiga de perra Beagle seccionada accidentalmente y reimplantada y suturada con patrón Gambee
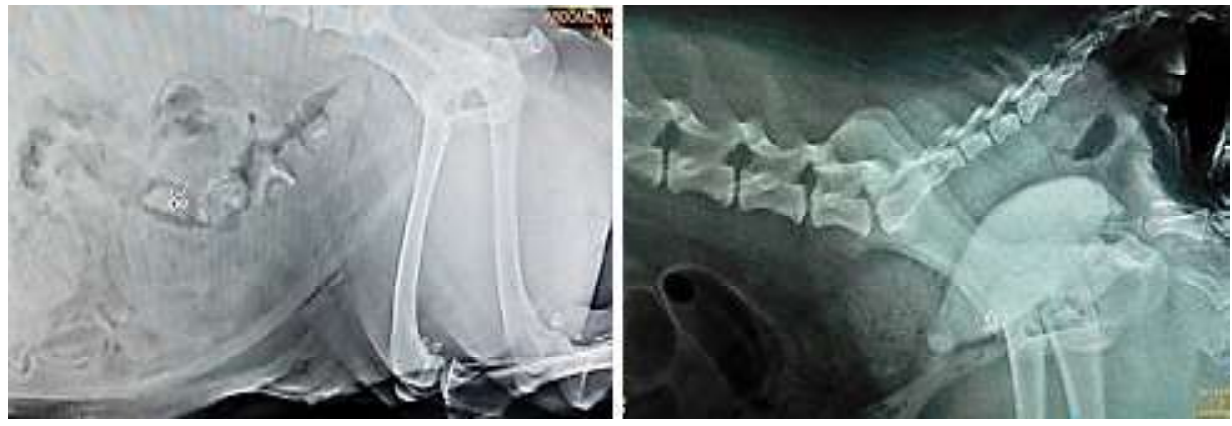

Figura 3. Radiografía pos-quirúrgica en perra Beagle con vejiga urinaria reimplantada. Ver sonda uretral (Izquierda). Urografía excretora (Derecha)

10 días se realizó una urografía excretora con el fin de confirmar la funcionalidad de las vías urinarias (Figura 3).

\section{Discusión}

Los signos clínicos de piometra de cuello cerrado se caracterizan por ser inespecíficos, estando entre los más relevantes el vómito, inapetencia, letargo y deshidratación. Usualmente se llega al diagnóstico a través de las ayudas diagnósticas como ecografía, rayos X (Danova et al., 2012). Se conoce que las bacterias presentes en el útero pueden contaminar la cavidad abdominal cuando el útero friable se rompe, cuando las bacterias son capaces de atravesar la pared uterina inflamada y, cuando migran desde la luz uterina a través del tubo uterino hasta el ova- 
rio (Rubio et al., 2014). Los cambios en el hemograma pueden ir desde neutrofilia con desviación a la izquierda hasta leucopenia, anemia normocítica, normocrómica no regenerativa; además, en el perfil bioquímico se puede encontrar hiperproteinemia, hiperglobulinemia y azoemia (MacPhail y Fossum, 2018). En el presente caso se presentaron los cambios más comúnmente asociados a piometra como son leucocitosis por neutrofilia con desviación a la izquierda, aumento del nitrógeno ureico en sangre (BUN) y disminución de proteínas plasmáticas (Davidson, 2014; Hagman, 2018).

Entre las complicaciones más comúnmente asociadas a cirugía de vejiga urinaria se ha reportado disminución de la luz vesical, hematoma vesical, dehiscencia, denervación, incontinencia urinaria, fugas, uroperitoneo, daño en uréteres y, muerte (McLoughlin, 2019). La vejiga urinaria se puede suturar en una o dos capas, con patrón continuo o interrumpido. Thieman-mankin et al. (2012) no encontraron diferencias entre pacientes que fueron suturados en una o dos capas. En este caso, la vejiga suturó en una capa, con un patrón discontinuo, con el fin de lograr una adecuada confrontación de las capas y las paredes de la vejiga, y buscando una alternativa para la revascularización.

El objetivo principal en la reparación de la vejiga es lograr la regeneración de las tres capas, preservar su funcionalidad y capacidad de distensión. Para lograr esto, se ha experimentado con polímeros sintéticos de policaprolactona, ácido poli-L-láctico, ácido poliglicólico (Shakhssalim et al. 2010), implantación de aloinjerto de membrana amniótica canina (Sheta et al., 2014), entre otros. Un estudio demostró mediante histología que a las 10 semanas de la cirugía, la pared de la vejiga se encuentra regeneradas (Wongsetthachai et al., 2011), de manera que el aloinjerto o autoinjerto debe contemplarse cuando exista pérdida considerable de tejido vesical, como ocurre al extirpar neoplasias, tejido desvitalizado o defectos de inervación (Wongsetthachai et al., 2011). En el presen- te caso se realizó la reimplantación del cuerpo de la vejiga al cuello utilizando un patrón de sutura continuo en una sola capa, con sutura monofilamentosa no absorbible 3-0, según reporta la literatura (MacPhail y Fossum, 2018).

La complicación en este caso fue el daño en la inervación del músculo detrusor. Por ese motivo se decidió realizar la omentalización en el sitio de incisión y, de esa manera aprovechar la capacidad de control de infección, proveer hemostasia, angiogénesis y reinervación en los sitios donde se adhiere (Agner et al., 2001; Campbell, 2004).

Para medir la funcionalidad del sistema excretor se dispone de diversas pruebas imagenológicas (Rademacher, 2019). Para poder evaluar las vías urinarias altas, la mejor elección es la urografía excretora, la cual permite valorar la pelvis renal, los uréteres y la vejiga urinaria; asimismo, es la única que admite la visualización de la continuidad de las vías urinaria altas, necesaria en este caso para evidenciar la correcta reimplantación ureteral y vesical (Heuter, 2005). Para poder verificar una adecuada micción y evitar la incontinencia urinaria, se recomienda el uso de pruebas como la cistometría y el perfil de presión uretral, sin embargo, son pruebas costosas y requieren de equipos especiales (Goldstein y Westropp, 2005). Otra prueba eficaz y de menor costo para la evaluación de las vías urinarias bajas es la cistografía de contraste, la cual permite evaluar la continuad y lesiones murales de la vejiga urinaria (Essman, 2005). En este caso el paciente fue evaluado duante dos semanas demostrando una adecuada micción y continuidad del medio de contraste en la urografía excretora.

\section{Literatura Citada}

\section{Agner C, Yeomans D, Dujovny M. 2001.}

The neurochemical basis for the applications of the greater omentum in neurosurgery. Neurol Res 23: 7-15. doi: 10.1179/01616410-1101198226 
2. Campbell BG 2004. Omentalization of a nonresectable uterine stump abscess in a dog. J Am Vet Med Assoc 224: 17991803. doi: 10.2460/javma.2004.224.1799

3. Danova N, Schmiedt C, Bjorling D. 2012. Female reproductive system. In: Williams \& Niles (eds). Manual of canine and feline abdominal surgery. Barcelona, España: Lexus. p 385-411.

4. Davidson A. 2014. Clinical conditions of the bitch and queen. In: Small animal internal medicine. $6^{\text {th }}$ ed. St. Louis, Missouri; Elsevier. p 915-943.

5. Essman SC. 2005. Contrast cystography. Clin Tech Small An P 20: 46-51. doi: 10.1053/j.ctsap.2004.12.007

6. Gaut MM, Ortiz J. 2015. Management of abdominal compartment syndrome after transurethral resection of the prostate. Braz J Anesthesiol 65: 519-521. doi: 10.1016/j.bjane.2013.12.001

7. Goldstein RE, Westropp JL. 2005. Urodynamic testing in the diagnosis of small animal micturition disorders. Clin Tech Small An P 20: 65-72. doi: 10.1053/ j.ctsap.2004.12.009

8. Hagman R. 2018. Pyometra in small animals. Vet Clin N Am-Small 48: 639661. doi: 10.1016/j.cvsm.2018.03.001

9. Heuter KJ. 2005. Excretory urography. Clin Tech Small An P 20: 39-45. doi: 10.1053/j.ctsap.2004.12.006

10. Lipscomb V. 2018. Bladder. In: Johnston $\mathrm{S}$, Tobias K (eds). Veterinary surgery small animal. $2^{\text {nd }}$ ed. St. Louis, Missouri: Elsevier. p 2219-2233.

11. MacPhail C, Fossum T. 2018. Surgery of the bladder and urethra. In: Fossum T (ed). Small animal surgery. $5^{\text {th }}$ ed. Barcelona, España: Elsevier. p 678-719.

12. McLoughlin MA. 2011. Complications of lower urinary tract surgery in small animals. Vet Clin N Am-Small 41: 889913. doi: 10.1016/j.cvsm.2011.07.001

13. Muraro L, White RS. 2014. Complications of ovariohysterectomy procedures performed in 1880 dogs. Tierarztl
Prax K H 42: 297-302. doi: 10.1055/s0038-1623776

14. Rademacher N. 2019. Diagnostic imaging of the urinary tract. Vet Clin $\mathrm{N}$ Am-Small 49: 261-286. doi: 10.1016/ j.cvsm.2018.10.006

15. Rubio A, Boyen F, Tas O, Kitshoff A, Polis I, Van Goethem B, et al. 2014. Bacterial colonization of the ovarian bursa in dogs with clinically suspected pyometra and in controls. Theriogenology 82: 966-971. doi: 10.1016/j.theriogenology.2014.07.016

16. Shakhssalim N, Soleimani M, Dehghan MM, Rasouli J, Taghizadeh-Jahed M, Torbati PM, et al. 2017. Bladder smooth muscle cells on electrospun poly( $\varepsilon$-caprolactone)/ poly(L-lactic acid) scaffold promote bladder regeneration in a canine model. Mat Sci Eng C-Biomim 75: 877-884. doi: 10.1016/j.msec.2017.02.064

17. Sheta E, Elzomor S, Eltookhy O, Elkader NAA, Ahmed KA. 2014. Structural and functional renovation of urinary bladders after amniotic membrane implantation in dogs. Int J Vet Sci Med 2: 57-66. doi: 10.1016/j.ijvsm.2014.05.001

18. Thieman KM, Pozzi A. 2010. Torsion of the urinary bladder after pelvic trauma and surgical fixation. Vet Comp Orthopaed 23: 259-261. doi: 10.3415/ VCOT-09-10-0111

19. Thieman-mankin KM, Ellison GW, Jeyapaul CJ, Glotfelty-ortiz, CS. 2012. Comparison of short-term complication rates between dogs and cats undergoing appositional single-layer or inverting double-layer cystotomy closure: 144 cases (1993-2010). J Am Vet Med Assoc 240: 65-68. doi: 10.2460/javma.240.1.65

20. Wongsetthachai P, Pramatwinai $C$, Banlunara W, Kalpravidh M. 2011. Urinary bladder wall substitution using autologous tunica vaginalis in male dogs. Res Vet Sci 90: 156-159. doi: 10.1016/ j.rvsc.2010.05.015 